\title{
Congruency effects can compensate for deficits of healthy older adults in crossmodal integration
}

Focko L. Higgen ${ }^{1}{ }$, Charlotte Heine ${ }^{1}$, Lutz Krawinkel ${ }^{1}$ MD, Florian Göschl ${ }^{2}$ PhD, Andreas K. Engel ${ }^{2}$ MD, $^{2}$ Friedhelm C. Hummel ${ }^{3,4,5}$ MD, Gui Xue ${ }^{6}$ PhD, Christian Gerloff ${ }^{1}$ MD

${ }^{1}$ Department of Neurology, University Medical Center Hamburg-Eppendorf, 20246 Hamburg, Germany

${ }^{2}$ Department of Neurophysiology and Pathophysiology, University Medical Center Hamburg-

Eppendorf, 20246 Hamburg, Germany

${ }^{3}$ Defitech Chair of Clinical Neuroengineering, Brain Mind Institute and Center for Neuroprosthetics, Swiss Federal Institute of Technology (EPFL), Geneva, Switzerland

${ }^{4}$ Defitech Chair of Clinical Neuroengineering, Brain Mind Institute and Center for Neuroprosthetics, Swiss Federal Institute of Technology Valais (EPFL Valais), Clinique Romande de Réadaptation, Sion, Switzerland

${ }^{5}$ Clinical Neuroscience, Medical School University of Geneva, Geneva, Switzerland

${ }^{6}$ State Key Laboratory of Cognitive Neuroscience and Learning, Beijing Normal University, Beijing, China, 100875

Short Title: Crossmodal integration in healthy older adults

\author{
*Corresponding Author \\ Focko Lorenz Higgen \\ Department of Neurology \\ University Medical Center Hamburg-Eppendorf
}


bioRxiv preprint doi: https://doi.org/10.1101/673491; this version posted June 19, 2019. The copyright holder for this preprint (which was not certified by peer review) is the author/funder. All rights reserved. No reuse allowed without permission.

\section{Martinistraße 52}

20246 Hamburg, Germany

Tel: $+49-40-7410-55573$

Fax: $+49-40-7410-57391$

E-mail: f.higgen@uke.de

Keywords: aging, multisensory, rehabilitation, tactile, visual 


\section{Abstract}

2 One of the pivotal challenges of aging is to maintain independence in the activities of daily life. In

3 order to adapt to changes in the environment, it is crucial to continuously process and accurately

4 combine simultaneous input from different sensory systems, i.e., crossmodal integration.

5 With aging, performance decreases in multiple cognitive domains. The processing of sensory stimuli

6 constitutes one of the key features of this deterioration. Age-related sensory impairments affect all

7 modalities, substantiated by decreased acuity in visual, auditory or tactile detection tasks.

8 However, whether this decline of sensory processing leads to impairments in crossmodal integration

9 remains an unresolved question. While some researchers propose that crossmodal integration degrades with age, others suggest that it is conserved or even gains compensatory importance.

11 To address this question, we compared behavioral performance of older and young participants in a well-established crossmodal matching task, requiring the evaluation of congruency in simultaneously presented visual and tactile patterns. Older participants performed significantly worse than young controls in the crossmodal task when being stimulated at their individual unimodal visual and tactile perception thresholds. Performance increased with adjustment of stimulus intensities. This improvement was driven by better detection of congruent stimulus pairs $(p<0.01)$, while detection of incongruent pairs was not significantly enhanced $(p=0.12)$. scenarios and demanding cognitive tasks. Performance is enhanced when inputs to the visual and tactile systems are congruent. Congruency effects might therefore be used to develop strategies for cognitive training and neurological rehabilitation. 


\section{Introduction}

As the percentage of older people in the population increases, aging related declines gain more and more significance. An important agenda therefore is to identify means for supporting older adults to maintain sound minds and independent living.

In order to behave adequately in our natural environment, it is crucial to continuously process simultaneous input from different sensory systems and integrate this information to meaningful percepts (1). This crossmodal integration complements unimodal sensory perception and allows for basing decisions and behavior on a broader range of sensory cues (2).

Age-related decline affects processes of crossmodal integration in several ways. The processing of sensory stimuli constitutes one of the key features of this deterioration. Age-related sensory impairments affect all modalities, as mirrored in decreased acuity in visual, auditory or tactile detection tasks (3-8). Apart from a decline of peripheral sensory organs, older adults show deficits in attention, working memory, episodic memory, decision making and subsequent actions (9-11). These cognitive domains are highly reliant on the crossmodal perception of information and vice versa.

However, the relevance of crossmodal integration in older adults is still under debate (see for example 12-15). While some authors report that the neurocomputational integration of multiple sensory stimuli degrades with age (e.g. 16-18), others suggest that crossmodal integration is conserved or even gains compensatory importance in older adults (e.g. 19-22).

Classically, the decline in sensory organs and higher cognitive domains was thought to prevent older adults from taking advantage of crossmodal information, by restricting effective multisensory integration processes and limiting the cognitive resources needed (e.g. 18).

More recent evidence, however, points to enhanced crossmodal integration in older adults. Different mechanisms have been discussed as possible reasons for this enhancement. Freiherr and colleagues (15) argue that crossmodal information can compensate for the age-related impairments of peripheral sensory organs. According to the principle of inverse effectiveness $(23,24)$ crossmodal integration can lead to maximal behavioral enhancement during perception of weak or ambiguous stimuli, due to appropriate weighting of the sensory evidence. Consequently, the integration of multiple sensory inputs could ameliorate unimodal decline in older adults' sensory systems and improve the overall outcome of perceptual processing (25).

Age-related alterations in central neurocomputational processes have been discussed to influence crossmodal integration. General cognitive slowing in older adults, demonstrated in several tasks (26- 
28), has been suggested to lead to more susceptibility to crossmodal integration in older adults by extending the temporal window for possible crossmodal interactions $(29,30)$. Furthermore, it has been proposed that gains in performance in scenarios with crossmodal stimulation $(31,32) \mathrm{might}$ relate to increases in baseline crossmodal interactions in older adults due to neural noise $(33,34)$. To further characterize the relevance of crossmodal integration in older adults, we investigated group differences between healthy older and young participants in a well-established visuo-tactile matching task (35-38). In this task, participants have to evaluate congruency in simultaneously presented visual and tactile dot patterns. Most studies that found a behavioral benefit of older adults in crossmodal tasks have focused on visual-auditory integration (20-22). Data on other sensory modalities are sparse. Our study focuses on visuo-tactile integration, as there is evidence suggesting that tasks involving interaction of visual and somatosensory stimuli profit strongly from crossmodal interactions effects $(39,40)$. In everyday life, the tactile modality interacts mainly with vision to recognize our surroundings and facilitate orientation. The latter represents a basic ability needed for interacting with the environment and to preserve independence $(41,42)$.

To be able to compare participants' performance and subjective task difficulty across both groups and modalities, we determined individual unimodal perception thresholds prior to the crossmodal study $(43,44)$.

Our first hypothesis was that unimodal perception thresholds for visual and tactile pattern recognition should be higher in the older group compared to young, due to multiple age-related sensory impairments (45). Second, we hypothesized that older participants would show greater enhancement of performance compared to young in a crossmodal integration task involving stimuli presented at the individual unimodal perceptual thresholds - in accordance with the more recent notion in the field.

\section{Materials and Methods}

\section{Participants}

37 older and 22 young volunteers were screened for the study. Six older volunteers did not meet the inclusion criteria during initial assessment. One older and two young participants dropped out because of personal reasons or technical problems. Ten older participants (five female, mean $(\mathrm{M})=$ 74.1 years, standard deviation $(S D)=3.90$ years) did not meet the predefined accuracy criterion in a training session prior to the threshold estimation (described in detail in 'Experimental procedure') and were no longer considered in the analysis. Thus, the final sample consisted of 20 young (11 female, $M=24.05$ years, $S D=2.50$ ) and 20 older ( 11 female, $M=72.14$ years, $S D=4.48$ ) volunteers. 
86

All participants were right-handed according to the Edinburgh handedness inventory (Oldfield, 1971), had normal or corrected to normal vision, no history or symptoms of neuro-psychiatric disorders (MMSE $\geq 28$, DemTect $\geq 13$ ) and no history of centrally acting drug intake. All participants received monetary compensation for participation in the study.

\section{Assessment}

Prior to inclusion, each participant underwent an assessment procedure. Assessment consisted of a neurological examination, the Mini-Mental State Examination (MMSE; 46) and the DemTect (47) to rule out symptoms of neuro-psychiatric disorders. Furthermore, a 2-point-discrimination test (cut-off $>3 \mathrm{~mm} ; 48,49$ ) and a test of the mechanical detection threshold (cut-off $>0.75 \mathrm{mN}$; MDT, v. Frey Filaments, OptiHair2-Set, Marstock Nervtest, Germany; 50,51) were conducted to ensure intact peripheral somatosensation.

\section{Setup and stimuli}

The experiment was conducted in preparation for a magnetoencephalography (MEG) study and the setup was designed to match conditions in the MEG laboratory. The experiment took place in a lightattenuated chamber. We chose experimental procedure, stimulus configuration and stimulation parameters based on pilot data showing accuracy of tactile pattern recognition to be very different between older and young participants.

We used an adapted version of a well-established experimental paradigm, the visuo-tactile matching task (35-38). Participants are instructed to compare tactile patterns presented to the right index fingertip and visual patterns presented on a computer screen. For tactile stimulation, the participants' right hand was resting on a custom-made board containing a Braille stimulator (QuaeroSys Medical Devices, Schotten, Germany, see Figure 1). The Braille stimulator consists of eight pins arranged in a four-by-two matrix, each $1 \mathrm{~mm}$ in diameter with a spacing of $2.5 \mathrm{~mm}$. Each pin is controllable independently. Pins can be elevated for any period of time to form different patterns. At the end of each pattern presentation, all pins return to baseline. The stimuli consisted of four geometric patterns, each of them formed by four elevated pins (Figure 1). Participants passively perceived the elevated pins without active exploration. A 15 -inch screen at $60 \mathrm{~Hz}$ with a resolution of $1024 \times 768$ pixels, positioned $65 \mathrm{~cm}$ in front of the participants, served for presentation of the visual stimuli. The design of the visual patterns was analogous to the tactile patterns. Visual patterns had a size of $43 \times 104$ pixels. They were presented left of a central fixation point on a noisy background (Perlin noise; Figure 1D). 
117 Depending on the task, the amplitude of pin elevation and the grey intensity of visual patterns were

118 adjusted, while the duration of the pattern presentation was always kept constant at $500 \mathrm{~ms}$. The

119 amplitude of the pin elevation can be controlled in 4095 discrete steps, with a maximum amplitude

120 of $1.5 \mathrm{~mm}$. Maximal grey intensity equaled black patterns with RGB: 0-0-0.

121 We used Presentation software (Neurobehavioral Systems, version 15.1) to control stimulus

122 presentation and to record participants' response time (RT) and accuracies.

\section{Experimental procedure}

124 All participants who met the predefined accuracy criterion in a training session prior to the 125 experiment (at least 75\% correct answers in a tactile-to-visual delayed match-to-sample task with 126 easy tactile patterns) performed a series of tasks representing the current experiment (tactile 127 habituation, tactile threshold estimation, visual habituation, visual threshold estimation, unimodal 128 training, matching task; see Figure 1B). At the beginning of each task participants read the task 129 instructions presented on a computer screen. The experiment started with the tactile habituation 130 task.

131 Tactile habituation. The tactile habituation task consisted of a tactile-to-visual delayed match-to132 sample task. Four target patterns were introduced as the stimulus set (see Figure 1B, trial sequence), 133 at maximum pin amplitude and with a duration of $500 \mathrm{~ms}$. Each trial started with a central white 134 fixation point appearing on a noisy background. This fixation point remained visible throughout each 135 trial. The tactile pattern presentation started $1500 \mathrm{~ms}$ after appearance of the fixation point with a 136 stimulus chosen pseudo-randomly from the stimulus set. After the tactile presentation and a waiting 137 interval of $1200 \mathrm{~ms}$, the central fixation point turned into a question mark and participants indicated 138 which of the four patterns had been presented. Participants responded via button press with the 139 fingers two to five of the left hand. After each trial participants received visual feedback (1000ms) 140 whether their response was correct (green ' + ') or incorrect (red '-'; see Figure 1C). The waiting 141 interval after stimulus offset was integrated to prepare for the following MEG experiment, where it 142 allowed for avoiding motor artefacts in the MEG signal. The background changed after every trial. 143 After a minimum of five training blocks, each consisting of 16 trials, and an accuracy of at least $75 \%$ in 144 three of five consecutive blocks, participants could proceed to the next step. If participants did not 145 reach the target accuracy within 15 blocks, they were excluded from further participation.

146 Tactile threshold estimation. Pilot studies indicated that most older adults were able to recognize the 147 target patterns at 500ms stimulus presentation time in the unimodal tactile condition with an 148 accuracy of approximately $80 \%$ correct. However, using the same parameters, young performed 
149 close to $100 \%$. Equally, visual recognition accuracy was close to $100 \%$ in both groups for these

150 parameters. To achieve a comparable performance of around $80 \%$ correct answers for both

151 modalities in older and young participants, we conducted an adaptive staircase procedure to detect

152 thresholds for visual and tactile pattern recognition and tailor stimulus intensities for each

153 participant.

154 Since the slope of the psychometric function was supposed to be very different in older and young

155 participants and we did not have any priors regarding the exact shape, we decided not to use a

156 Bayesian approach (e.g. Quest; 52), but to implement a non-parametric adaptive staircase procedure

157 (53-56). We designed a two-down/one-up fixed-step-size adaptive staircase. With a ratio $\Delta$-down/ $\Delta$ -

158 up $=0.5488$, this staircase should converge around $80.35 \%$. For tactile pattern presentation,

159 adaptation of the height of the braille pins rendered recognition easier or more complicated. Step

160 size was determined after piloting with approximately $0.1 \mathrm{~mm}$ up, $0.055 \mathrm{~mm}$ down. The staircase

161 started with the maximum amplitude of $1.5 \mathrm{~mm}$. The staircase stopped after 20 reversals, while

162 proceeding at boundary levels. The last 16 reversals served to calculate thresholds. Participants

163 performed this staircase for both unimodal visual and unimodal tactile stimulation. Trial timing was

164 the same as in the habituation task, except there was no feedback given.

165 Visual habituation. The visual habituation task followed the same procedure as in the tactile 166 condition. Instead of tactile stimulation, patterns were presented visually at maximal contrast (see

167 Figure 1C, target patterns). Again, participants continued to fixate the central point during pattern

168 presentation, so that visual patterns would appear in the left visual hemi-field. Trial timing, block

169 design and accuracy criterion were the same as for the tactile recognition task.

170 Visual threshold estimation. The visual threshold estimation followed the same procedure as in the

171 tactile modality. For visual threshold estimation, adaptation of the grey intensity of the pattern

172 varied the patterns' contrast against the noisy background. Step size was determined after piloting,

173 with a step up being two intensities, and a step down one on the grey scale ranging from 47 (RGB:

174 138-138-138) to 101 (RGB: 0-0-0). The staircase started with the maximum contrast (black pattern;

175 RGB: 0-0-0). Pilot data showed that a grey intensity of RGB: 138-138-138, which corresponds to the

176 mean of the grey values of our noisy background, was hardest to detect. Therefore, this contrast was

177 the lower boundary of the staircase. Trial timing and stimulus duration remained the same as in the

178 tactile threshold estimation process.

179 Following the threshold estimation in tactile and visual modalities, participants performed a short

180 unimodal training in both conditions to verify thresholds calculated from the adaptive staircase

181 procedure. The order of modalities was chosen randomly. Trial timing remained the same as in the 
182 habituation tasks. To keep performance at a comparable level, thresholds were adjusted if accuracy

183 was below $75 \%$ or above $85 \%$ over 5 blocks. For the adjustment, the same step sizes as in the

184 adaptive staircase were used.

185 Visuo-tactile matching. After the unimodal threshold estimation, participants conducted the visuo-

186 tactile matching task. In this task, visual and tactile patterns were presented with synchronous onset

187 and offset, and participants had to decide whether the patterns were congruent or incongruent.

188 Participants responded with the left index ('congruent') or middle finger ('incongruent') via button

189 press on a response box and again visual feedback (a green ' + ' or a red '-') was given in every trial.

190 Trial timing was the same as in the unimodal recognition task (Figure 1C). Congruent and incongruent

191 stimulus pairs were presented equally often. Participants started the visuo-tactile matching task at

192 the stimulus intensity of the unimodal thresholds and performed a set of five consecutive blocks,

193 consisting of eight trials. If participants did not reach an average accuracy between $75 \%$ and $85 \%$

194 correct within these five blocks, stimulus intensities in both modalities were adapted according to

195 the steps of the adaptive staircase procedure.

196 Visual and tactile stimulus intensities were adjusted evenly. After adjustment of stimulus intensities

197 participants performed another set of five blocks. The experiment ended when participants reached

198 a stable performance between $75-85 \%$ correct averaged over a set of five blocks (mean number of

199 sets $=2.25$, standard deviation $=0.93$ ).

200 Statistical analysis

201 Statistical analyses were performed using Matlab (Version 8.4.0.150421, MathWorks, Natick, MA,

202 2014) and RStudio (Version 3.5.4, R Core Team, 2017).

203 To test for baseline group differences a multivariate analysis of variance (MANOVA) was performed

204 by means of R's manova() command to investigate the relationship between the values for sex, MDT,

205 2-point-discrimination, MMSE, DemTect as dependent variables and group (young group vs. older

206 group) as the independent variable. As group allocation was defined by participants' age, age was not

207 included into the model. For post-hoc analysis, two-sampled t-tests were performed and Benjamini-

208 Yekutieli (BY) correction was applied to adjust for multiple comparisons (57). To compare the older

209 and young groups' performance in the habituation tasks, another MANOVA was performed.

210 Dependent variables were the response accuracies in tactile and visual pattern recognition. For post-

211 hoc analysis, two-sampled t-tests were performed and BY correction was applied to adjust for

212 multiple comparisons. Another MANOVA was performed to compare accuracies and thresholds

213 before and after the unimodal training and between the two groups, whereby accuracies, grey 
214 intensities and pin heights were dependent variables and time (before vs. after the training) and

215 group (young group vs. older group) independent variables. Two-sample t-tests and BY correction

216 were used for post-hoc analysis of significant group effects.

217 As in the course of the visuo-tactile matching task pin height and grey intensity were adjusted evenly

218 according to the steps of the adaptive staircases, changes in stimulus intensities were highly

219 dependent. We therefore did not use MANOVA for further analyses. Two sample t-tests and BY

220 correction were used to compare accuracies and stimulus intensities between the groups in the first

221 and last set of five blocks of the visuo-tactile matching task. In addition, paired t-tests and BY

222 correction were performed to compare accuracies and stimulus intensities within the groups

223 between the first and the last set of the visuo-tactile matching task.

224 To evaluate detection performance for congruent and incongruent stimulus pairs, paired t-tests were

225 used to compare accuracies within and two-sampled t-tests to compare accuracies between groups.

226 BY correction was used to adjust for multiple comparisons.

- Figure 1 here -

\section{$228 \quad$ Results}

\section{Baseline data}

230 The group comparison of baseline data obtained in the assessment prior to inclusion (Table 1)

231 showed significant differences between the groups of young and older participants (Pillai's

232 Trace $=0.43, F=5.15, d f=(1,38), p<0.01)$. Post-hoc comparison of the baseline data showed that

233 DemTect scores $(p<0.001)$ differed significantly between groups. Importantly, the measurements

234 revealed age-appropriate, not pathological results in the older group.

\begin{tabular}{|c|c|c|}
\hline Metrics & Young group (n=20) & Older group (n=20) \\
\hline DemTect & $17.8( \pm 0.6)^{*}$ & $15.9( \pm 1.5)^{*}$ \\
\hline MMSE & $29.7( \pm 0.6)$ & $29.6( \pm 0.6)$ \\
\hline 2-Point $(\mathrm{mm})$ & $2.1( \pm 0.2)$ & $2.2( \pm 0.4)$ \\
\hline MDT (mN) & $0.28( \pm 0.1)$ & $0.57( \pm 0.5)$ \\
\hline
\end{tabular}


237 Mean values are shown \pm standard deviation. Based on a significant main effect of the factor group

238 (young group vs. older group), post-hoc tests were conducted. * indicate significant difference

239 between young and older participants, $p$-value $\leq 0.01$.

Habituation tasks

242 The analysis of performance in the habituation tasks revealed significant associations between

243 accuracy and group (Pillai's Trace $=0.41, F=12.65, d f=(1,38), p<0.001)$. Post-hoc comparison showed

244 that the young participants $(95.70 \pm 5.10 \%)$ performed significantly better in the tactile task

245 compared to the older group $(82.47 \pm 10.44 \%),(F(38)=25.93, p<0.001)$. In the visual task, response

246 accuracies did not differ between the groups (Young group: $98.12 \pm 2.94 \% / O l d e r$ group $98.12 \pm$

$247 \quad 2.94 \% ; F(38)=0, p=1)$.

\section{Threshold estimation and unimodal training}

249 The results of the threshold estimation are displayed in Figure 2.

250 Visual threshold estimation in the young group resulted in a mean grey intensity of $49.2 \pm 1.1$. The

251 mean adaptive staircase for tactile threshold estimation in the young group showed a course similar

252 to the visual condition and resulted in a mean threshold, i.e., pin height of $0.60 \pm 0.17 \mathrm{~mm}$.

253 Visual threshold estimation in the older group resulted in a mean grey intensity of $53.9 \pm 2.5$. The

254 mean adaptive staircase for tactile threshold estimation in the older group showed only a small

255 downward trend, indicating that the tactile threshold in the older group was close to maximum

256 stimulus intensity. The mean tactile threshold in the older group was $1.13 \pm 0.28 \mathrm{~mm}$.

257 To ensure the validity of the estimated thresholds, the unimodal training was performed. Within the

258 groups, there was no significant change of visual or tactile thresholds in the course of the training

259 (Pillai's Trace $=0.68, F=38.51, d f=(1,77), p=0.981)$, indicating a reliable threshold estimation. Across

260 groups, there was no difference in detection accuracy (tactile $F(77)=4.29, p=0.116$, visual $F(77)=0.72$,

$261 p=0.825)$, but as expected in pin height $(F(77)=112.86, p<0.001)$ and grey intensity $(F(77)=104.77$,

$262 p<0.001)$.

\section{Visuo-tactile matching}

265 The mean accuracies, tactile (pin heights) and visual (grey intensities) stimulus intensities of the first

266 and last set of five blocks of the visuo-tactile matching task were compared within and between

267 groups (Table 2). 
In the first set, older participants performed significantly worse $(t(37.98)=4.16, p<0.01)$ despite their significantly higher unimodal stimulus intensities (grey intensity: $t(19.17)=156.69, p<0.001$; pin height: $t(30.58)=-7.70, p<0.001)$. To reach a performance of around $80 \%$ correct responses in the older group, visual and tactile intensities had to be further increased significantly according to the steps of the adaptive staircase (visual: $t(19)=-6.71, p<0.001$, tactile: $t(19)=-7.75, p<0.001)$. With this adjustment of stimulus intensities task performance was significantly improved $(t(18)=-4.59, p<0.01)$ and there was no longer a significant difference in accuracy between the young and the older group $(t(36.86)=1.16, p=0.92)$. Within the young group, there was no difference between the first and the last set in accuracy $(t(19)=-0.55, p=1)$, grey intensity $(t(19)=0.81, p=1)$ and pin height $(t(19)=2.37$, $p=0.12)$

\begin{tabular}{|c|c|c|}
\hline & Young group $(n=20)$ & Older group $(n=20)$ \\
\hline \multicolumn{3}{|c|}{ First set of matching task } \\
\hline Accuracy (\%) & $78.31( \pm 9.09)^{*}$ & $66.20( \pm 9.31)^{* \#}$ \\
\hline Pin height (mm) & $0.58( \pm 0.17)^{*}$ & $1.14( \pm 0.28)^{* \#}$ \\
\hline Grey intensity & $49( \pm 1.38)^{*}$ & $53.65( \pm 2.70)^{* \#}$ \\
\hline \multicolumn{3}{|c|}{ Last set of matching task } \\
\hline Accuracy (\%) & $79.50( \pm 5.94)$ & $77.28( \pm 6.00)^{\#}$ \\
\hline Pin height $(\mathrm{mm})$ & $0.57( \pm 0.17)^{*}$ & $1.24( \pm 0.27)^{* \#}$ \\
\hline Grey intensity & $48.9( \pm 1.59)^{*}$ & $56.2( \pm 2.69)^{* \#}$ \\
\hline
\end{tabular}

Tab. 2. First and last set of the visuo-tactile matching task.

Mean values are shown \pm standard deviation for accuracy, grey intensity and pin height in the first and last set of the task, sorted by group. * indicate significant difference between young and older participants, all p-values $\leq 0.001 ;{ }^{*}$ indicate significant difference within the older group, all p-values $\leq 0.01$.

Congruent vs. incongruent stimulus pairs 
287 To further explore the differences in performance in the visuo-tactile matching task, we analyzed

288 detection accuracy for congruent and incongruent stimulus pairs separately.

289 Both age groups exhibited strong congruency effects with the detection rate for congruent patterns

290 being significantly higher than for incongruent pairs over all matching blocks (older group: congruent

$29182.12 \%$, incongruent $62.21 \%, t(19)=7.81, p<0.001$; young group: congruent $87.34 \%$, incongruent

$29270.33 \%, t(19)=5.51, p<0.001)$. While overall detection accuracy was significantly lower in the older

293 group in the first set of the visuo-tactile matching task (see 3.4), the difference in detection accuracy

294 for congruent vs. incongruent stimulus pairs was the same (18\%) for both age groups (older group

$29575.48 \%$ vs. $57.11 \%, p<0.01$ /young group $87.05 \%$ vs. $69.30 \%, p<0.01$; no difference between groups in

296 percentage difference, $p=0.88$; see Figure 3 ).

297 Adjustment of stimulus intensities increased mean accuracy of pattern detection in the older group

298 (see 3.4). Analyses for congruent vs. incongruent stimulus pairs showed that this effect was driven by

299 better detection of congruent patterns. With increased stimulus intensity, there was a significant

300 increase in the detection of congruent stimulus pairs $(t(17)=-3.48, p<0.01)$, while detection of

301 incongruent pairs was not significantly enhanced $(t(17)=-2.04, p=0.12)$. Due to this asymmetric

302 benefit, the congruency effect increased to $23 \%(88.12 \%$ vs. $65.47 \%, p<0.001$; see Figure 3D) in the

303 last set of the matching task in the older group.

- Figure 3 here -

\section{Discussion}

306 This study aimed to investigate performance differences in crossmodal integration between young and healthy older adults. The data show that older participants performed worse in unimodal tactile pattern recognition and had higher unimodal detection thresholds. The main finding was that in the crossmodal condition older participants showed higher thresholds compared to the unimodal condition, while young participants showed a stable performance. However, the performance of older participants could be enhanced by further increasing stimulus intensity. This effect was driven by higher detection rates for congruent stimulus pairs, while detection of incongruent pairs was not significantly enhanced. These data support the concept of an increased relevance of congruency effects in older adults in crossmodal tasks.

315 Confirming our first hypothesis, older participants showed significantly higher thresholds for 316 unimodal tactile and visual pattern recognition than the young. This is in line with previous findings 317 and most likely caused by age-related decline of sensory organs $(32,39)$. The data indicate that 
318 healthy older adults are able to perform at a comparable level of accuracy but require higher

319 stimulus intensities (58).

320 Contrary to our second hypothesis, the data showed that in the older group required stimulus

321 intensities were significantly higher for crossmodal pattern matching compared to the unimodal

322 conditions. Young participants performed significantly better in the crossmodal task at the

323 individually defined perception thresholds. Required stimulus intensities in the young group did not

324 differ between the unimodal and the crossmodal condition. However, even in the complex visuo-

325 tactile matching task older participants were able to reach the same level of performance as in the

326 unimodal detection task. This enhancement of performance with increased stimulus intensities was

327 driven by better detection of congruent stimulus pairs, while detection of incongruent stimuli did not

328 improve, resulting in a numerically stronger congruency effect $(23 \%)$ than in young adults $(18 \%)$.

329 Our data suggest that the previously observed benefit of crossmodal integration in older adults is not

330 necessarily driven by the crossmodal nature of the task but, rather, by congruency of the stimulus

331 materials $(21,22)$. It has been shown that stimulus congruence in crossmodal stimulation can have

332 great impact on behavior compared to unimodal stimulation. The so called 'congruency effect' is

333 thought to facilitate cognitive processing and to counteract age-related unimodal shortcomings

$334(39,59,60)$. This congruency effect has also been shown to apply for crossmodal congruent vs.

335 incongruent information perceived through various modalities $(21,36)$. Our data show congruency

336 effects for congruent vs. incongruent stimulation in young and older participants. At the individual

337 unimodal perception thresholds, congruency effects in the crossmodal task are similar in size in the

338 young and the older group. When performance levels are adjusted, congruency effects drive the

339 improved results in older participants in the crossmodal integration task.

340 Opposing our initial hypothesis, we did not find evidence for a simple enhancement of crossmodal

341 integration in older participants. There are different reasons that might account for these findings.

342 Earlier studies indicated that older adults do not benefit from crossmodal stimulation in very

343 complex tasks involving sensory as well as higher-order cognitive processes $(15,16,32)$. Laurienti et al.

344 (2006) found greater performance gains in an older compared to a young group in the crossmodal vs.

345 the unimodal condition. In contrast to our task, they used unequivocal stimulus material, namely

346 colored dots presented on a computer screen and (spoken) color words presented via loud speakers.

347 Poor performance of older participants in our visuo-tactile matching task might be due to increased

348 complexity and task demands of the crossmodal matching compared to unimodal pattern detection.

349 In addition to the complexity of the stimulus material, participants had to pay attention to visual and

350 tactile stimulation concurrently and identify patterns separately in both modalities before comparing 
351 them. This might be seen as a worst-case scenario for crossmodal integration. Integration of the two

352 stimuli is not facilitated in a bottom-up manner but has to be actively controlled.

353 As cognitive top-down-control mechanisms such as attention tend to decline with age and lead to

354 processing difficulties of incoming stimuli (11), this might be another reason for the poor

355 performance of older participants in the crossmodal condition. In line with this, the matching task

356 can also be viewed as a working memory task, as mental representations of patterns have to be

357 compared with each other. The ability to hold information in memory while manipulating it

358 decreases with age (10). Thus, decline of working memory capacity might be another reason for the

359 poor performance of older participants. Taken together, the mechanisms that are thought to lead to

360 enhanced crossmodal integration in older adults, such as the increase of baseline noise (32), general

361 cognitive slowing (29) or inverse effectiveness associated with sensory deficits (15) do not seem to

362 apply in our experimental setting which might be considered a scenario with rather complex task

363 demands.

364 In summary, older participants performed worse in a complex visuo-tactile matching task at the

365 individual perception thresholds. We do not find behavioral evidence for a simple, compensatory

366 enhancement of crossmodal integration in the older group. However, even in this complex task older

367 participants were able to perform at a comparable level with young adults when higher stimulus

368 intensities were offered. The relative improvement in performance after this adjustment of stimulus

369 intensities was driven by better detection of congruent stimulus pairs. The behavioral benefit of

370 congruent stimulus material in this complex crossmodal scenario underlies the importance of

371 congruency effects.

372 These findings might have implications for future applications of crossmodal tasks and scenarios.

373 Paying attention to more than one modality and basing one's decision on a wider range of cues has

374 been suggested to compensate for impaired unisensory processing (25). Destructive consequences of

375 sensory deterioration caused by cognitive and physiological changes are thought to be alleviated this

376 way. Following this idea, Laurienti et al. (2006) suggested the use of crossmodal everyday life gadgets

377 and multisensory training strategies for older adults. The current results add certain limitations to

378 the idea of crossmodal integration as a compensation mechanism for age-related impairments.

379 These limitations include the type and familiarity of stimuli and cognitive demands of a task. Complex

380 cognitive tasks seem to lower the older adults' capacity to compensate impairments. In this context,

381 the observed benefit of congruent stimulus material might be exploited in future studies and

382 practical applications. To use effects of crossmodal integration in everyday life, congruent

383 information with high stimulus intensities should be delivered through the target modalities. As one 
384 of the most important endeavors in this field is to identify means to support older adults to maintain 385 mental health and independent living, congruency effects might be one asset to help older adults 386 master cognitively demanding tasks or to cope with complex scenarios. Congruency effects might 387 also be used to develop strategies for care of disabled older adults as, for example, in neurological 388 rehabilitation.

389 There are some limitations to the current work. Due to high task demands, there was a high dropout 390 rate in the older group. Therefore, the older participants in our study represented a rather well 391 performing subpopulation. In a more population based older group, the found effects might even be 392 larger. Furthermore, variance in the older participants' performance was larger compared to the 393 young group. Heterogeneity in older adults is likely to occur with respect to sensory and cognitive impairments. Moreover, highly relevant behavioral and physiological changes not only occur from young to old, but also in higher age (e.g. 7). Considering these aspects, other studies divided participants into young, young-old and old-old. This approach offers the advantage of a more detailed view on the evolution of age-related changes and differences within the older population. Future studies investigating effects of crossmodal integration in older adults might consider recruiting more than two groups.

400 There are limitations to the current work. In the data presented here, variance in the older participants' performance was larger compared to the young group. Heterogeneity in older adults is likely to occur with respect to sensory and cognitive impairments. Moreover, highly relevant behavioral and physiological changes occur between the age of 65 and 80 years, merged as one 'older group' in the current study (e.g. Poliakoff et al., 2006). Considering these aspects, other studies divided participants into young, young-old and old-old. This approach offers the advantage of a more detailed view on the evolution of age-related changes and differences within the older population. Future studies investigating effects of crossmodal integration in older adults might consider 


\section{Statement of Ethics}

411 The study was conducted in accordance with the Declaration of Helsinki and was approved by the

412 local ethics committee of the Medical Association of Hamburg (PV5085). All participants gave written

413 informed consent.

\section{Disclosure Statement}

415 The authors have no conflicts of interest to declare.

\section{$416 \quad$ Funding Sources}

417 This work was funded by the German Research Foundation (DFG) and the National Science

418 Foundation of China (NSFC) in project Crossmodal Learning, SFB TRR169/A3/B1/B4 and by the

419 German Research Foundation (DFG) in project SFB 936/A3/C1/Z1. 
bioRxiv preprint doi: https://doi.org/10.1101/673491; this version posted June 19, 2019. The copyright holder for this preprint (which was not certified by peer review) is the author/funder. All rights reserved. No reuse allowed without permission.

\section{Author Contributions}

421 FLH: study design, data acquisition, data analyses, interpretation, preparation of manuscript. $\mathrm{CH}$ : 422 data acquisition, data analyses, interpretation, preparation of manuscript. LK: study design,

423 interpretation, revision of manuscript. FG: study design, interpretation, revision of manuscript. AKE: 424 study idea, interpretation, revision of manuscript. FCH: study idea, revision of manuscript GX: study 425 idea, interpretation, revision of manuscript. CG: study idea, study design, interpretation, revision of 426 manuscript. 


\section{References}

1. Calvert GA. Crossmodal processing in the human brain: insights from functional neuroimaging studies. Cereb Cortex. 2001 Dec;11(12):1110-23.

2. Calvert GA, Spence C, Stein BE. The Handbook of Multisensory Processing. 2004 [cited 2018 Dec 7]; Available from: https://researchportal.bath.ac.uk/en/publications/the-handbook-ofmultisensory-processing

3. Davis A, McMahon CM, Pichora-Fuller KM, Russ S, Lin F, Olusanya BO, et al. Aging and Hearing Health: The Life-course Approach. Gerontologist. 2016 Apr;56 Suppl 2:S256-267.

4. Jackson GR, Owsley C. Visual dysfunction, neurodegenerative diseases, and aging. Neurol Clin. 2003 Aug;21(3):709-28.

5. Kalina RE. Seeing into the future. Vision and aging. West J Med [Internet]. 1997 Oct [cited 2019 Jan 25];167(4):253-7. Available from: https://www.ncbi.nlm.nih.gov/pmc/articles/PMC1304540/

6. Kenshalo DR. Somesthetic sensitivity in young and elderly humans. J Gerontol. 1986 Nov;41(6):732-42.

7. Poliakoff E, Ashworth S, Lowe C, Spence C. Vision and touch in ageing: Crossmodal selective attention and visuotactile spatial interactions. Neuropsychologia [Internet]. 2006 [cited 2016 May 4];44(4):507-17. Available from: http://www.sciencedirect.com/science/article/pii/S0028393205002575

8. Wickremaratchi MM, Llewelyn JG. Effects of ageing on touch. Postgrad Med J. 2006 May;82(967):301-4.

9. Anguera JA, Gazzaley A. Dissociation of motor and sensory inhibition processes in normal aging. Clin Neurophysiol. 2012 Apr;123(4):730-40.

10. Gazzaley A, Cooney JW, Rissman J, D'Esposito M. Top-down suppression deficit underlies working memory impairment in normal aging. Nat Neurosci. 2005 Oct;8(10):1298-300.

11. Guerreiro MJS, Anguera JA, Mishra J, Van Gerven PWM, Gazzaley A. Age-equivalent top-down modulation during cross-modal selective attention. J Cogn Neurosci. 2014 Dec;26(12):2827-39.

12. Cienkowski KM, Carney AE. Auditory-visual speech perception and aging. Ear and hearing. 2002;23(5):439-449.

13. McGovern DP, Roudaia E, Stapleton J, McGinnity TM, Newell FN. The sound-induced flash illusion reveals dissociable age-related effects in multisensory integration. Front Aging Neurosci. 2014;6:250.

14. Setti A, Finnigan S, Sobolewski R, McLaren L, Robertson IH, Reilly RB, et al. Audiovisual temporal discrimination is less efficient with aging: an event-related potential study. Neuroreport. 2011 Aug 3;22(11):554-8.

15. Freiherr J, Lundström JN, Habel U, Reetz K. Multisensory integration mechanisms during aging. Front Hum Neurosci. 2013;7:863. 
16. Sommers MS, Tye-Murray N, Spehar B. Auditory-visual speech perception and auditory-visual enhancement in normal-hearing younger and older adults. Ear Hear. 2005 Jun;26(3):263-75.

17. Stephen JM, Knoefel JE, Adair J, Hart B, Aine CJ. Aging-related changes in auditory and visual integration measured with MEG. Neurosci Lett. 2010 Oct 22;484(1):76-80.

18. Stine EA, Wingfield A, Myers SD. Age differences in processing information from television news: the effects of bisensory augmentation. J Gerontol. 1990 Jan;45(1):P1-8.

19. Diaconescu AO, Hasher L, Mclntosh AR. Visual dominance and multisensory integration changes with age. Neuroimage. 2013 Jan 15;65:152-66.

20. Diederich A, Colonius $\mathrm{H}, \mathrm{Schomburg} A$. Assessing age-related multisensory enhancement with the time-window-of-integration model. Neuropsychologia. 2008;46(10):2556-2562.

21. Laurienti PJ, Burdette JH, Maldjian JA, Wallace MT. Enhanced multisensory integration in older adults. Neurobiol Aging. 2006 Aug;27(8):1155-63.

22. Peiffer AM, Mozolic JL, Hugenschmidt CE, Laurienti PJ. Age-related multisensory enhancement in a simple audiovisual detection task. Neuroreport. 2007 Jul 2;18(10):1077-81.

23. Meredith MA, Stein BE. Visual, auditory, and somatosensory convergence on cells in superior colliculus results in multisensory integration. J Neurophysiol. 1986 Sep;56(3):640-62.

24. Meredith MA, Stein BE. Interactions among converging sensory in puts in the superior colliculus. Science. 1983 Jul 22;221(4608):389-91.

25. Hairston WD, Laurienti PJ, Mishra G, Burdette JH, Wallace MT. Multisensory enhancement of localization under conditions of induced myopia. Exp Brain Res. 2003 Oct;152(3):404-8.

26. Birren JE, Fisher LM. Aging and speed of behavior: possible consequences for psychological functioning. Annu Rev Psychol. 1995;46:329-53.

27. Cerella J. Information processing rates in the elderly. Psychol Bull. 1985 Jul;98(1):67-83.

28. Salthouse TA. Aging and measures of processing speed. Biol Psychol. 2000 Oct;54(1-3):35-54.

29. Setti A, Stapleton J, Leahy D, Walsh C, Kenny RA, Newell FN. Improving the efficiency of multisensory integration in older adults: audio-visual temporal discrimination training reduces susceptibility to the sound-induced flash illusion. Neuropsychologia. 2014 Aug;61:259-68.

30. Verhaeghen P, De Meersman L. Aging and the Stroop effect: a meta-analysis. Psychol Aging. 1998 Mar;13(1):120-6.

31. Hugenschmidt CE, Mozolic JL, Laurienti PJ. Suppression of multisensory integration by modalityspecific attention in aging. Neuroreport. 2009 Mar 4;20(4):349-53.

32. Mozolic JL, Hugenschmidt CE, Peiffer AM, Laurienti PJ. Multisensory Integration and Aging. In: Murray MM, Wallace MT, editors. The Neural Bases of Multisensory Processes [Internet]. Boca Raton (FL): CRC Press/Taylor \& Francis; 2012 [cited 2016 Apr 5]. (Frontiers in Neuroscience). Available from: http://www.ncbi.nIm.nih.gov/books/NBK92841/ 
33. Hugenschmidt CE, Mozolic JL, Tan H, Kraft RA, Laurienti PJ. Age-related increase in crosssensory noise in resting and steady-state cerebral perfusion. Brain Topogr. 2009 May;21(34):241-51.

34. Voytek B, Knight RT. Dynamic network communication as a unifying neural basis for cognition, development, aging, and disease. Biol Psychiatry. 2015 Jun 15;77(12):1089-97.

35. Göschl F, Friese U, Daume J, König P, Engel AK. Oscillatory signatures of crossmodal congruence effects: An EEG investigation employing a visuotactile pattern matching paradigm. Neuroimage. 2015 Aug 1;116:177-86.

36. Göschl F, Engel AK, Friese U. Attention modulates visual-tactile interaction in spatial pattern matching. PLOS ONE. 2014;9(9):e106896.

37. Hummel FC, Gerloff $\mathrm{C}$. Interregional long-range and short-range synchrony: a basis for complex sensorimotor processing. Prog Brain Res. 2006;159:223-36.

38. Wang $P$, Göschl $F$, Friese $U$, König $P$, Engel AK. Long-range functional coupling predicts performance: Oscillatory EEG networks in multisensory processing. Neuroimage. $2019 \mathrm{Apr}$ 5;196:114-25.

39. Mahoney JR, Li PCC, Oh-Park M, Verghese J, Holtzer R. Multisensory integration across the senses in young and old adults. Brain Res. 2011 Dec 2;1426:43-53.

40. Misselhorn J, Daume J, Engel AK, Friese U. A matter of attention: Crossmodal congruence enhances and impairs performance in a novel trimodal matching paradigm. Neuropsychologia. 2015 Jul 21;

41. Oie KS, Kiemel T, Jeka JJ. Multisensory fusion: simultaneous re-weighting of vision and touch for the control of human posture. Brain Res Cogn Brain Res. 2002 Jun;14(1):164-76.

42. Tipper SP, Lloyd D, Shorland B, Dancer C, Howard LA, McGlone F. Vision influences tactile perception without proprioceptive orienting. Neuroreport. 1998 Jun 1;9(8):1741-4.

43. Beer AL, Röder B. Unimodal and crossmodal effects of endogenous attention to visual and auditory motion. Cogn Affect Behav Neurosci. 2004 Jun;4(2):230-40.

44. Venkatesan UM, Festa EK, Ott BR, Heindel WC. Differential Contributions of Selective Attention and Sensory Integration to Driving Performance in Healthy Aging and Alzheimer's Disease. J Int Neuropsychol Soc. 2018 May;24(5):486-97.

45. Mancini DJ, Allen S. Geriatric Physiology. In: Pignolo RJ, Ahn J, editors. Fractures in the Elderly: A Guide to Practical Management [Internet]. Cham: Springer International Publishing; 2018. p. 65-78. Available from: https://doi.org/10.1007/978-3-319-72228-3_4

46. Folstein MF, Folstein SE, McHugh PR. "Mini-mental state". A practical method for grading the cognitive state of patients for the clinician. J Psychiatr Res. 1975 Nov;12(3):189-98.

47. Kalbe E, Kessler J, Calabrese P, Smith R, Passmore AP, Brand M, et al. DemTect: a new, sensitive cognitive screening test to support the diagnosis of mild cognitive impairment and early dementia. Int J Geriatr Psychiatry. 2004 Feb;19(2):136-43. 
48. Crosby PM, Dellon AL. Comparison of two-point discrimination testing devices. Microsurgery. 1989;10(2):134-7.

49. Dellon ES, Keller K, Moratz V, Dellon AL. The relationships between skin hardness, pressure perception and two-point discrimination in the fingertip. J Hand Surg Br. 1995 Feb;20(1):44-8.

50. Fruhstorfer H, Gross W, Selbmann O. von Frey hairs: new materials for a new design. Eur J Pain. 2001;5(3):341-2.

51. Rolke R, Magerl W, Campbell KA, Schalber C, Caspari S, Birklein F, et al. Quantitative sensory testing: a comprehensive protocol for clinical trials. Eur J Pain. 2006 Jan;10(1):77-88.

52. Watson AB, Pelli DG. QUEST: a Bayesian adaptive psychometric method. Percept Psychophys. $1983 \mathrm{Feb} ; 33(2): 113-20$.

53. García-Pérez MA. Forced-choice staircases with fixed step sizes: asymptotic and small-sample properties. Vision Res. 1998 Jun;38(12):1861-81.

54. Kaernbach C. Simple adaptive testing with the weighted up-down method. Percept Psychophys. 1991 Mar;49(3):227-9.

55. Treutwein B. Adaptive psychophysical procedures. Vision Res. 1995 Sep;35(17):2503-22.

56. Wetherill GB, Levitt H. SEQUENTIAL ESTIMATION OF POINTS ON A PSYCHOMETRIC FUNCTION. Br J Math Stat Psychol. 1965 May;18:1-10.

57. Benjamini Y, Yekutieli D. The Control of the False Discovery Rate in Multiple Testing under Dependency. The Annals of Statistics [Internet]. 2001 [cited 2018 Aug 23];29(4):1165-88. Available from: https://www.jstor.org/stable/2674075

58. Humes LE, Busey TA, Craig JC, Kewley-Port D. The effects of age on sensory thresholds and temporal gap detection in hearing, vision, and touch. Atten Percept Psychophys. 2009 May;71(4):860-71.

59. Miller J. Timecourse of coactivation in bimodal divided attention. Percept Psychophys. 1986 Nov;40(5):331-43.

60. Miller J. Divided attention: evidence for coactivation with redundant signals. Cogn Psychol. 1982 Apr;14(2):247-79. 


\section{Figure Legends}

Fig. 1. Stimulus design and experimental procedure.

A: Braille stimulator. For tactile stimulation, the participants' right hand was resting on a custommade board containing the Braille stimulator (QuaeroSys Medical Devices, Schotten, Germany), with the fingertip of the right index finger placed above the stimulating unit. B: Sequence of tasks in the experiment, C: Stimuli consisted of four different patterns, D: After a pre-stimulus interval of $1500 \mathrm{~ms}$, tactile and/or visual patterns were presented for $500 \mathrm{~ms}$ depending on the current step of the experiment. After a wait interval of $1200 \mathrm{~ms}$, a question mark appeared on the screen and participants gave the response via button press. Depending on the current step of the experiment, visual feedback was given (1000ms).

Fig. 2. Summary of threshold estimations for visual and tactile stimulus intensities.

Graphs depict the mean stimulus intensity ( $y$-axis) per trial ( $x$-axis) during the course of the adaptive staircase over all participants (young group = blue; older group $=$ red) with standard deviations (hatched areas). Number of trials equals trials in shortest threshold estimation procedure, i.e. trials common to all participants. A: Visual threshold estimation in young participants, B: Tactile threshold estimation in young participants, C: Visual threshold estimation in older participants, D: Tactile threshold estimation in older participants.

\section{Fig. 3. Detection accuracy of congruent vs. incongruent stimulus pairs.}

Boxplots of detection accuracy for congruent vs. incongruent stimulus pairs for the two groups in the first and the last set of the matching task. A: Performance for the first set of the matching task in young participants, B: Same as A but for the last set of the matching task in young participants, C: Performance for the first set of the matching task in older participants, D: Same as C but for the last set of the matching task in older participants. 

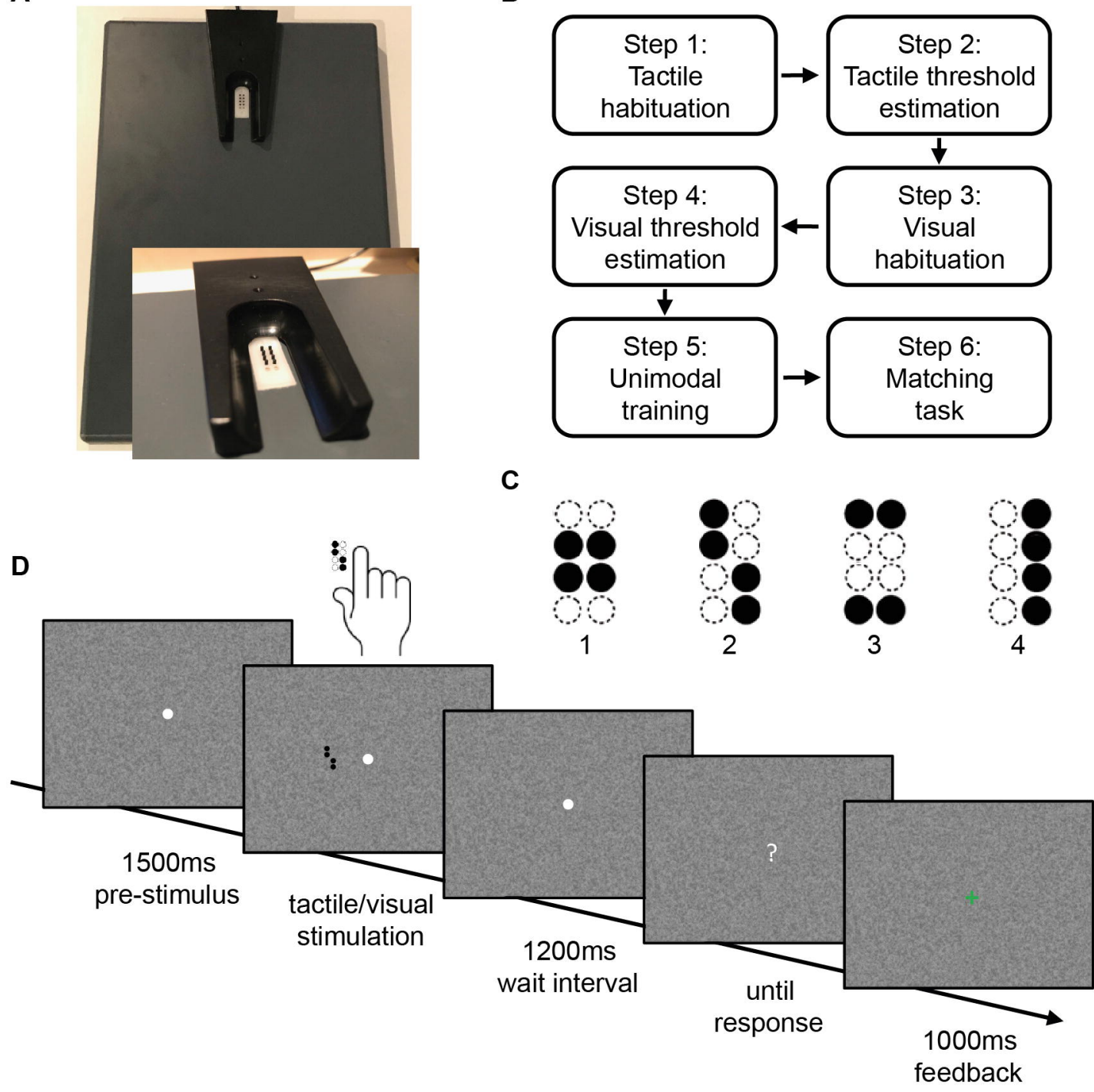
A

Visual threshold estimation in young participants

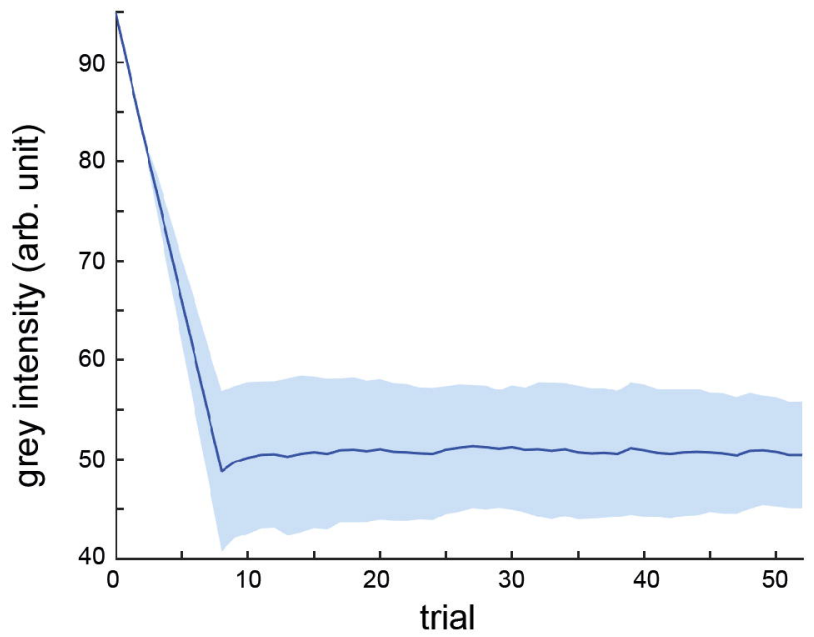

B

Tactile threshold estimation in young participants

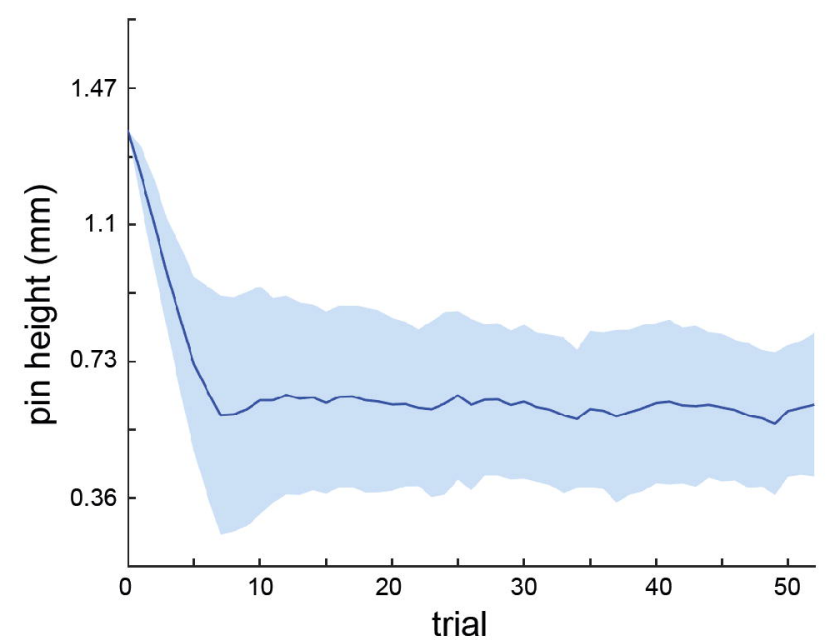

C

Visual threshold estimation in older participants

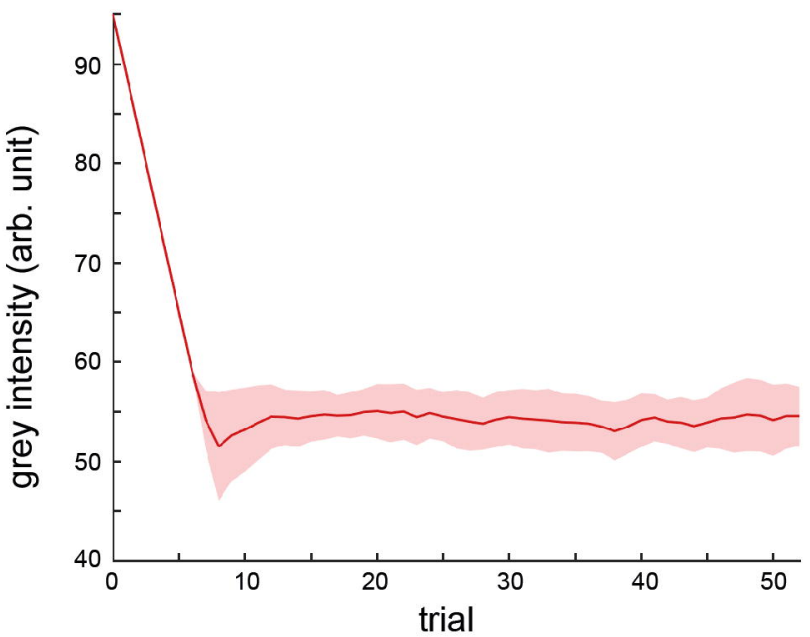

D

Tactile threshold estimation in older participants

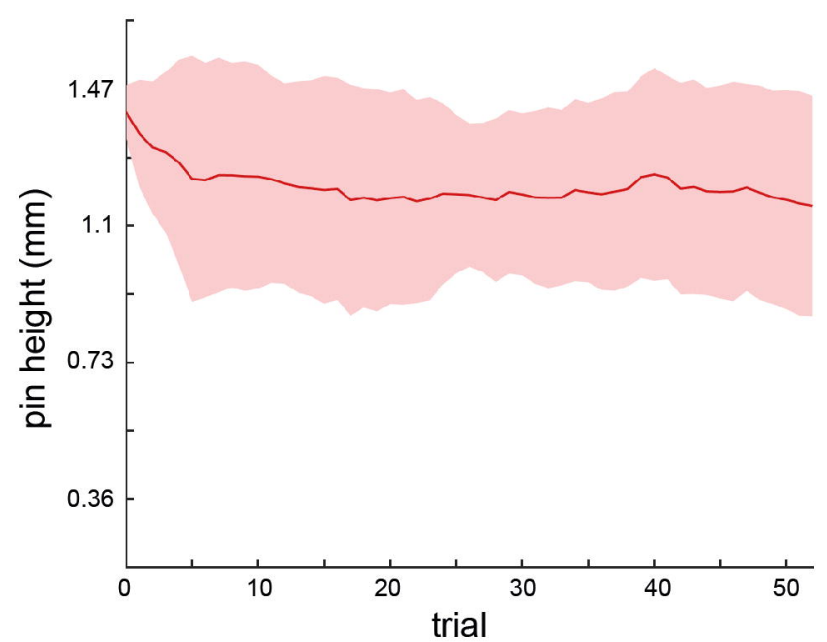


A First set matching task young group

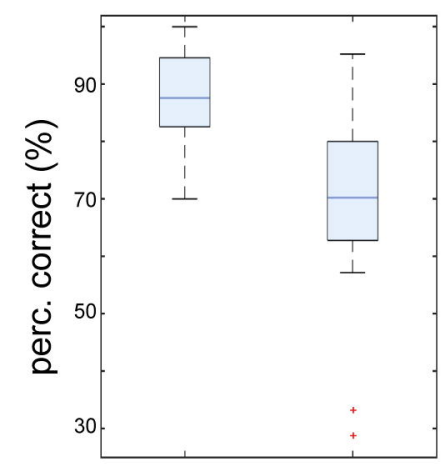

congruent incongruent
B Last set matching task young group

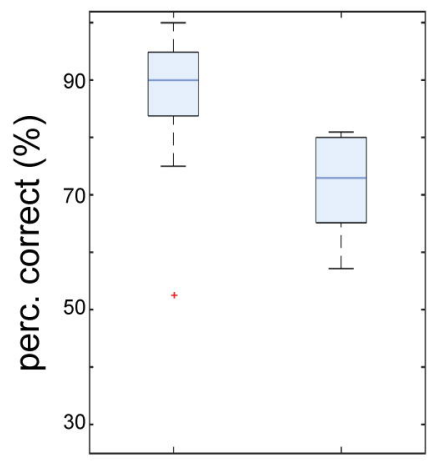

congruent incongruent
C First set matching task older group

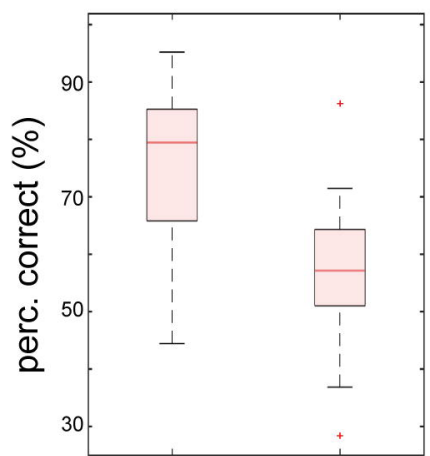

congruent incongruent
D Last set matching task older group

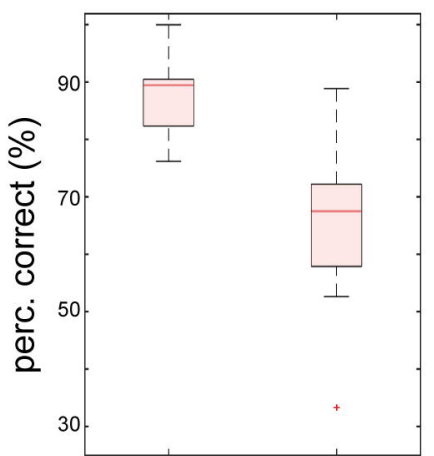

congruent incongruent 